correlation coefficient (ICC) between predicted and real values. Pearson's $r$ was calculated too. The free and open source programming language $\mathrm{R}$ was used for all the analyses. Dataset source: Borráz-León, Javier I. (2020), "Dark triad, attractiveness, mate value, and sexual partners", Mendeley Data, V1, doi: 10.17632/87vx6jfnrp.1

Results: Machiavellianism, narcissism and psychopathy predictions obtained ICC of 0.593, 0.335, 0.505 and Pearson's $r$ of $0.608,0.346,0.548$ respectively. The results were indicative of fair performance, mainly for Machiavellianism and psychopathy.

Conclusions: AI might be useful to predict MNP. This could be utile in many situations, such as dealing with missing data or deciding whether to formally test someone. Finally, the AI used in this study is freely available, allowing anyone to experiment.

Disclosure: No significant relationships.

Keywords: Artificial Intelligence; Personality; traits; psychometry

\section{Posttraumatic stress disorder}

\section{O205}

The psychiatric impact of the 2020 beirut port explosion on civilians and relief workers

\section{R. Charara* and J. El-Khoury}

Psychiatry, American University of Beirut Medical Center, Beirut, Lebanon

${ }^{\star}$ Corresponding author.

doi: 10.1192/j.eurpsy.2021.387

Introduction: On August 4th 2020, a massive port explosion shook Beirut, killing at least 200, injuring more than 6,000 people and leaving more than a quarter of a million living in unfit homes. Various factors can participate in the severity of mental health outcomes of a disaster including the number of injuries, the degree of property destruction, unexpectedness of the occurrence of the event, and the type of the disaster.

Objectives: The main aim of this study is to assess the prevalence of post-traumatic stress disorder (acute stress disorder) and major depression at 1 and 6 months following the Beirut explosion. The secondary aim is to determine predictors of PTSD incidence among civilians and relief workers affected by the disaster.

Methods: This is a cross-sectional study with data collected via an online survey through convenience sampling. People will be recruited via social media platforms. To achieve a power of $80 \%$ and a two-sided significance of $5 \%$ and because gender differences will be explored, assuming a design effect (deff) of 2.5 , a minimum sample of 960 participants would be needed. The survey will include sociodemographic data, questions about exposure levels to trauma and a psychiatric symptom inventory. Pearson's Chi Square test will be used to examine the association between categorical variables and regression models will be run to examine the associations while controlling for confounders, including age, gender and others.

Results: The results from both rounds of data collection (months 1 and 6) will be available in late March 2021.

Conclusions: to follow based on results

Disclosure: No significant relationships.

Keywords: ptsd; community mental health; trauma

\section{6}

Post-traumatic stress, anxiety and depression after intensive care unit stay: Findings from a general hospital

\author{
U. Bhaumik $^{1_{\star}}$, V. Subramaniyam ${ }^{2}$ and R. Kandukuru ${ }^{3}$ \\ ${ }^{1}$ Psychiatry, Independent Practice, Kolkata, India; ${ }^{2}$ Psychiatry, \\ Independent practice, Bangalore, India and ${ }^{3}$ Anaesthesiology And \\ Critical Care, M S Ramaiah Medical College, Bangalore, India \\ ${ }^{\star}$ Corresponding author.
}

doi: 10.1192/j.eurpsy.2021.388

Introduction: Post-traumatic stress disorder (PTSD) following intensive care is a relatively new entity. It is triggered due to traumatic experiences in a setting of threat to life due to illness. Prolonged stay in intensive care predisposes to delusional memories related to the stay experience and may increase likelihood of post-traumatic stress.It may also present as anxiety or depression. Objectives: This study explored the prevalence of post-traumatic symptoms in intensive care, find its correlates and its impact on health-related quality of life (HRQoL).

Methods: 225 adult patients admitted for at least 1 day in the intensive care unit (ICU) of a general hospital in Bangalore,India were recruited and assessed at 1 week, 1 month and 3 months after ICU discharge. Subjects were assessed for ICU related memories, PTSD, anxiety and depression scores and quality of life at and post discharge.

Results: $59.6 \%$ of the study population had significant posttraumatic stress, including anxiety in $62.35 \%$, depression in $10.58 \%$ and mixed anxiety-depression in $27.06 \%$. Delusional memories were found in $31.6 \%$. Presence of delusional memories was found to have significant correlation with post-traumatic stress and had a negative impact on HRQoL.

Conclusions: This study was the first of its kind from Asia. More systematic studies on PTSD following ICU stay and its correlates are required as available evidence lacks homogeneity. Suitable preventive measures should be taken to reduce prevalence of post-traumatic stress in intensive care due to its lasting impact on HRQoL.

Disclosure: No significant relationships.

Keywords: post-traumatic stress disorder; anxiety and depression; delusional memories; intensive care unit

\section{O207}

A comparison of mdma-assisted psychotherapy to non-assisted psychotherapy in treatment-resistant PTSD: A systematic review and meta-analysis

B. Illingworth ${ }^{1 \star}$, D. Lewis ${ }^{2}$, A. Lambarth ${ }^{3}$, K. Stocking ${ }^{4}$, J. Duffy ${ }^{5}$, L. Jelen ${ }^{6}$ and J. Rucker ${ }^{6}$

${ }^{1}$ Respiratory Medicine, Cambridge University Hospitals NHS Foundation Trust, Cambridge, United Kingdom; ${ }^{2}$ Acute Medicine, Brighton \& Sussex University Hospitals NHS Trust, Brighton, United Kingdom; ${ }^{3}$ Clinical Pharmacology, University College London

Hospitals NHS Foundation Trust, London, United Kingdom; ${ }^{4}$ Centre For Biostatistics, University of Manchester, Manchester, United Kingdom; ${ }^{5}$ Institute For Women's Health, University College London, London, United Kingdom and ${ }^{6}$ Centre For Affective Disorders, Institute of Psychiatry, Psychology \& Neuroscience, King's College London, London, United Kingdom

${ }^{\star}$ Corresponding author.

doi: 10.1192/j.eurpsy.2021.389 
Introduction: Novel, evidence-based treatments are required for treatment-resistant post-traumatic stress disorder (PTSD). 3,4Methylenedioxymethamphetamine (MDMA) has beneficially augmented psychotherapy in several small clinical trials.

Objectives: To review the use of MDMA-assisted psychotherapy in treatment-resistant PTSD.

Methods: Systematic searches of four databases were conducted from inception to February 2020. A meta-analysis was performed on trials which were double-blinded, randomised, and compared MDMAassisted psychotherapy to psychotherapy and placebo. The primary outcomes were the differences in Clinician Administered PTSD Scale (CAPS-IV) score and Beck's Depression Inventory (BDI). Secondary outcome measures included neurocognitive and physical adverse effects, at the time, and within seven days of intervention.

Results: Four randomised controlled trials (RCTs) met inclusion criteria. When compared to active placebo, intervention groups taking 75mg (MD -46.90; 95\% CI -58.78, -35.02), $125 \mathrm{mg}$ (MD -20.98; 95\% CI -34.35, -7.61) but not 100mg (MD -12.90; 95\% CI $-36.09,10.29$ ) of MDMA with psychotherapy, had significant decreases in CAPS-IV scores, as did the inactive placebo arm (MD -33.20; 95\% CI -40.53, -25.87). A significant decrease in BDI when compared to active placebo (MD -10.80; 95\% CI -20.39, -1.21 ) was only observed at $75 \mathrm{mg}$. Compared to placebo, participants reported significantly more episodes of low mood, nausea and jaw-clenching during sessions and lack of appetite after seven days. Conclusions: These results demonstrate potential therapeutic benefit with minimal physical and neurocognitive risk for the use of MDMA-assisted psychotherapy in TR-PTSD, despite little effect on Beck's Depression Inventory. Better powered RCTs are required to investigate further.

Disclosure: James Rucker has attended trial related meetings paid for by Compass Pathways Ltd.

Keywords: MDMA; ptsd; Treatment-resistance; psychotherapy

\section{O208}

Suicidality in post-traumatic stress disorder (PTSD) and complex PTSD (CPTSD)

L. Longo*, T. Jannini, M. Merlo, V. Cecora, M. Gagliano, B. D’Imperia, A. Daverio, L. Monaco, R. Rossi, C. Niolu, A. Siracusano and G. Di Lorenzo

Department Of Systems Medicine, University of Rome Tor Vergata, Rome, Italy

${ }^{\star}$ Corresponding author.

doi: 10.1192/j.eurpsy.2021.390

Introduction: International Classification of Diseases 11th Revision (ICD-11) has inserted complex post-traumatic stress disorder (cPTSD) as a clinically distinct disorder, different from PTSD. The diagnosis of $\mathrm{CPTSD}$ has the same requirements for the one of PTSD, in addition to disturbances of self-organization (DSO - e.g., disturbances in relationships, affect dysregulation, and negative selfconcept).

Objectives: This study aimed to explore suicidality in PTSD and cPTSD. We examined also the association between clinical dimensions of hopelessness (feelings, loss of motivation, future expectations) and other symptomatologic variables.

Methods: The sample, recruited at the Fondazione Policlinico Tor Vergata, Rome, Italy, consisted of 189 subjects, 132 diagnosed with
PTSD, and 57 with CPTSD, according to the ICD-11 criteria. Participants underwent the following clinical assessments: Clinician-Administered PTSD Scale (CAPS), Impact of Event Scale-Revised (IES), Beck Depression Inventory (BDI), Symptom Checklist-90-Revised (SCL-90), Dissociative Experience Scale (DES), Beck Hopelessness Scale (BHS).

Results: cPTSD showed significantly higher BHS-total $(\mathrm{p}=0.01)$ and BHS-loss of motivation subscale $(\mathrm{p}<0.001)$ scores than PTSD. Besides, cPTSD showed significantly higher scores in all clinical variables except for the IES-intrusive subscale. By controlling for the confounding factor "depression", suicidality in cPTSD (and in particular the BHS-total) appears to be correlated with IES-total score $(\mathrm{p}=0.042)$ and with DES-Absorption $(\mathrm{p}=0.02)$. Differently, no such correlations are found in PTSD.

Conclusions: Our study shows significant symptomatologic differences between PTSD and CPTSD, including suicidality. Indeed, suicidality in CPTSD appears to be correlated with the "loss of motivation" dimension, which fits well within the ICD-11 criteria of DSO.

Disclosure: No significant relationships.

Keywords: cPTSD; ptsd; Suicidality; Hopelessness

\section{O209}

Efficacy and safety results from the first pivotal phase 3 randomized controlled trial of mdma-assisted psychotherapy for treatment of severe chronic PTSD

\section{B. Van Der Kolk}

School Of Medicine, Boston University, West Stockbridge, United States of America

${ }^{*}$ Corresponding author. doi: 10.1192/j.eurpsy.2021.391

Introduction: Posttraumatic stress disorder is a prevalent mental health condition with substantial impact on daily functioning that lacks sufficient treatment options. Previous research has led to the designation of 3,4-methylenedioxymethamphetamine (MDMA) as a Breakthrough Therapy for treatment of post-traumatic stress disorder (PTSD) when administered as an adjunct to psychotherapy.

Objectives: Here we report the findings of the first randomized, double-blind, Phase 3 trial assessing the efficacy and safety of 3 sessions with a flexible dose of MDMA or placebo administered under direct observation to participants with severe PTSD $(n=100)$ as an adjunct to inner-directed psychotherapy.

Methods: Change in PTSD symptoms (CAPS-5) and functional impairment (SDS) were assessed by a central, blinded Independent Rater Pool at baseline and following each treatment session. Adverse events (AEs), concomitant medications, suicidal ideation and behavior were tracked throughout the study. Vital signs were measured during experimental sessions. The primary endpoint was 18 weeks post-randomization.

Results: Change in CAPS-5 and SDS, placebo-subtracted Cohen's d effect size, and a responder analysis will be presented. There were three serious AEs of suicidal ideation or behavior reported. MDMA was well tolerated, with some treatment emergent AEs occurring at greater frequency for the MDMA group during and after experimental sessions. 